Int. J. Electrochem. Sci., 13 (2018) $7816-7826$

\title{
Electrochemical immunosensor detection for lactoferrin in milk powder
}

\author{
Junyi Huang ${ }^{1,2}$, Ziyu He ${ }^{2, \#}$ Jie Cao $^{2}$, Jiang Hong ${ }^{2}$, Zhengjun $\mathrm{Wu}^{1, *}$, Haiyan Gao $^{2}$, Xianyan Liao $^{2, *}$ \\ ${ }^{1}$ State Key Laboratory of Dairy Biotechnology, Bright Dairy \& Food Co. Ltd., Shanghai 200072, \\ China; \\ ${ }^{2}$ Key Laboratory of Food Nutrition and Function, School of Life Sciences, Shanghai University, \\ Shanghai, 200444, China \\ ${ }^{\#}$ Co-first author: the contribution for this article is equal to Junyi Huang. \\ *E-mail: wuzhengjun@brightdairy.com,xyliao@shu.edu.cn
}

doi: $10.20964 / 2018.08 .47$

Received: 12 April 2018/ Accepted: 16 June 2018 / Published: 5 July 2018

\begin{abstract}
A lactoferrin (LF) immunosensor was fabricated by immobilizing an LF monoclonal antibody on a gold electrode, and the assembly process was tracked by cyclic voltammetric measurements and electrochemical impedance spectroscopy. A linear relationship between the immunosensor response current and the logarithm of the LF concentration was characterized to be in the range of 0.01-1000 ng $\mathrm{mL}^{-1}$ with a LF detection limit (LOD) of $4.9 \mathrm{pg} \mathrm{mL}^{-1}$. The fabricated LF immunosensor was specific to LF with no response to interfering substances. The optimum response occurred at an incubation temperature of $37{ }^{\circ} \mathrm{C}$ and an incubation time of $60 \mathrm{~min}$. In addition, the fabricated LF immunosensor exhibited a 4-week shelf life. Application of the fabricated LF immunosensor to real milk samples was demonstrated. In conclusion, we have developed a biosensor method for LF detection with high sensitivity and sufficient stability.
\end{abstract}

Keywords: Lactoferrin; Immunosensor; Bioelectrochemistry; Cyclic voltammetry

\section{FULL TEXT}

(C) 2018 The Authors. Published by ESG (www.electrochemsci.org). This article is an open access article distributed under the terms and conditions of the Creative Commons Attribution license (http://creativecommons.org/licenses/by/4.0/). 